\title{
STERILE NEUTRINOS: PHENOMENOLOGY AND THEORY
}

\author{
RABINDRA N. MOHAPATRA \\ Department of Physics, University of Maryland \\ College Park, MD 20742, USA \\ E-mail: rmohapat@physics.umd.edu
}

\begin{abstract}
A sterile neutrino in addition to the three known neutrinos seems unavoidable if one wants a simultaneous understanding of the results of the LSND experiment indicating $\nu_{\mu}-\nu_{e}$ oscillation together with other evidences for neutrino oscillations such as those from solar and atmospheric neutrino deficits. A major theoretical challenge then is to understand why the sterile neutrino is so light. A simple solution appears to be to assume that it is the lightest neutrino of a mirror sector of the universe which has identical matter and gauge content as the standard model. After a brief review of the phenomenology, a recently constructed realistic grand unified model based on the gauge group $S O(10) \times S O(10)^{\prime}$ that embeds the mirror universe idea is presented. Detailed predictions for known and the sterile neutrino sector are given and their consequences for cosmology are mentioned.
\end{abstract}

\section{Why we may need a sterile neutrino?}

The announcement by the Super-Kamiokande collaboration 1 of the evidence for neutrino oscillation (and hence nonzero neutrino mass) in their atmospheric neutrino data is a major milestone in the search for new physics beyond the standard model, which predicts that neutrinos are massless. In addition to the Super-Kamiokande atmospheric neutrino data, there are now strong indications for neutrino oscillations from the five solar neutrino experiments (Kamiokande, Homestake, Gallex, Sage and Super-Kamiokande2 3 ), other atmospheric neutrino observations 6 and the direct laboratory observation in the LSND experiment5. To explain all three experiments, three different scales for mass differences $\left(\Delta m^{2}\right)$ are needed. The atmospheric neutrino data requires $\Delta m_{\mu-X}^{2} \sim 3 \times 10^{-4}-7 \times 10^{-3} \mathrm{eV}^{2}$ whereas the solar neutrino data prefers either $3 \times 10^{-6}-7 \times 10^{-6} \mathrm{eV}^{2}$ or $\sim 10^{-10} \mathrm{eV}^{2}$ depending on whether the solution arises via the MSW mechanism or via oscillation in vacuum. The LSND data on the other hand prefers $0.3 \mathrm{eV}^{2} \leq \Delta m_{e \mu}^{2} \leq 2.0 \mathrm{eV}^{2}$ with $\Delta m^{2}$ as high as $10 \mathrm{eV}^{2}$ also in the allowed range. Since with the three known neutrinos one can get only two independent $\Delta m^{2}$ 's, it has been suggested 0 that a fourth sterile neutrino be invoked. For the uninitiated a sterile neutrino is defined as one whose interaction with standard model particles (such as the $W, Z$ etc) is much weaker than the strength of usual weak interaction. The reason for this is the discovery at LEP and SLC that only three neutrino species couple to the Z-boson. It is the goal of this talk to discuss

a possible theoretical scenario for the sterile neutrino after a brief discussion of how 
its introduction solves the neutrino puzzles.

\section{Scenarios for solving the neutrino puzzles}

In the presence of this extra neutrino species $\left(\nu_{s}\right)$, one can construct several scenarios for solving the three neutrino puzzles 6 6. the sterile neutrino to solve the neutrino puzzles 6 , it was proposed that the solar neutrino puzzle is solved via the oscillation of the $\nu_{e}$ to $\nu_{s}$ using the MSW mechanism 10 and the atmospheric neutrino puzzle is solved via the $\nu_{\mu}-\nu_{\tau}$ oscillation with maximal mixing. The solar neutrino puzzle fixes the $\Delta m_{e-s}^{2} \simeq(0.35-.75) \times 10^{-5} \mathrm{eV}^{2}$, whereas the atmospheric neutrino puzzle implies that $\Delta m_{\mu-\tau}^{2} \simeq 10^{-3} \mathrm{eV}^{2}$. This gives a picture where the $\nu_{s}$ and $\nu_{e}$ are close by in mass with nearly zero mass and the $\nu_{\mu}$ and $\nu_{\tau}$ are nearly degenerate in mass. The masses of the $\nu_{\mu}-\nu_{\tau}$ system is determined by the LSND experiment $m_{\nu_{2}} \simeq m_{\nu_{3}} \simeq \sqrt{\Delta m_{L S N D}^{2}}$. If the universe has a hot component in its dark matter, as some recent analyses suggest11 , then this requires the $\nu_{\mu}$ and $\nu_{\tau}$ masses to be each 2-3 eV implying that in LSND and KARMEN12 one should observe a $\Delta m^{2} \simeq 4-9 \mathrm{eV}^{2}$.

This scenarion is testable in the SNO 13 experiment once they measure the solar neutrino flux $\left(\Phi_{\nu}^{N C}\right)$ in their neutral current data and compare with the corresponding charged current value $\left(\Phi_{\nu}^{C C}\right)$. If the solar neutrinos convert to active neutrinos, then one would expect $\Phi_{\nu}^{C C} / \Phi_{\nu}^{N C} \simeq .5$, whereas in the case of conversion to sterile neutrinos, the above ratio would be nearly $\simeq 1$.

A second scenario advocated in Ref 8 and in 6 suggests that it is the atmospheric $\nu_{\mu}$ 's that oscillate into the sterile neutrinos whereas the solar neutrino oscillation could be involving either active or sterile ones depending on how many sterile neutrinos one postulates. The present atmospheric neutrino data cannot distinguish between the $\nu_{\tau}$ and $\nu_{s}$ as the final oscillation products. There is however an interesting suggestion 14 that monitoring the pion production via the neutral current reaction $\nu_{\tau}+N \rightarrow$ $\nu_{\tau}+\pi^{0}+N$ (which is absent in the case of sterile neutrinos) can help in distinguishing between these two possibilities.

A possible mass matrix for the first case is 15 :

$$
M=\left(\begin{array}{cccc}
\mu_{1} & \mu_{3} & 0 & 0 \\
\mu_{3} & 0 & 0 & \epsilon \\
0 & 0 & \delta & m \\
0 & \epsilon & m & \pm \delta
\end{array}\right) .
$$

Solar neutrino data requires $\mu_{3} \ll \mu_{1} \simeq 2 \times 10^{-3} \mathrm{eV}$ and $\epsilon \simeq .05 \mathrm{~m}$. In the case with the negative sign in the 44-entry, the $\Delta m^{2}$ in the atmospheric data as well as the mixing in the LSND oscillation are linked to one another. An interesting prediction of this mass matrix is that barring extreme fine tuning of parameters, the effective mass measured in neutrinoless double beta decay would have an upper bound $<m_{\nu}>\leq 2 \times 10^{-3} \mathrm{eV}$. 
Finally, it has recently been pointed out 16 that the introduction of the sterile neutrino seems to alleviate the problems connected with understanding of the heavy element (elements beyond $F e$ ) nucleosynthesis by r-processes around the supernovae. This may be taken as an independent argument for the introduction of sterile neutrinos regardless of the situation in the neutrino oscillation observations.

Finally, one has to ensure that all the new light particles introduced to explain the sterile neutrino do not spoil the success of big bang nucleosynthesis which cannot tolerate more than 1.5 extra neutrinos 17 . The contribution of a sterile neutrino is governed by its mass difference -squared and mixing with the normal neutrinos. For instance, the contribution of a sterile neutrino is suppressed 18 as long as the following inequality is satisfied:

$$
\Delta m^{2} \sin ^{4} 2 \theta \leq 3 \times 10^{-6} \quad e V^{2}
$$

Any theoretical model must respect this constraint.

\section{Mirror universe theory of the sterile neutrino}

If the existence of the sterile neutrino becomes confirmed say, by a confirmation of the LSND observation of $\nu_{\mu}-\nu_{e}$ oscillation or directly by SNO neutral current data to come in the early part of the next century, a key theoretical challenge will be to construct an underlying theory that embeds the sterile neutrino along with the others with appropriate mixing pattern, while naturally explaining its ultralightness.

It is clear that if a sterile neutrino was introduced into the standard model, the gauge symmetry does not forbid a bare mass for it implying that there is no reason for the mass to be small. It is a common experience in physics that if a particle has mass lighter than normally expected on the basis of known symmetries, then it is an indication for the existence of new symmetries. This line of reasoning has been pursued in recent literature to understand the ultralightness of the sterile neutrino by using new symmetries beyond the standard model.

We will focus on the recent suggestion that the ultralightness of the $\nu_{s}$ may be related to the existence of a parallel standard model19,80 which is an exact copy of the known standard model (i.e. all matter and all gauge forces identical). The mirror sector of the model will then have three light neutrinos which will not couple to the Z-boson and would not therefore have been seen at LEP. We will call the $\nu_{i}^{\prime}$ as the sterile neutrinos of which we now have three. The lightness of $\nu_{i}^{\prime}$ is dictated by the mirror $B^{\prime}-L^{\prime}$ symmetry in a manner parallel to what happens in the standard model. The two "universes" communicate only via gravity or other forces that are equally weak. This leads to a mixing between the neutrinos of the two universes and can cause neutrino oscillation between $\nu_{e}$ of our universe to $\nu_{e}^{\prime}$ of the parallel one in order to explain for example the solar neutrino deficit.

At an overall level, such a picture emerges quite naturally in superstring theories which lead to $E_{8} \times E_{8}^{\prime}$ gauge theories below the Planck scale with both $E_{8}$ s connected 
by gravity. We hasten to emphasize that despite this apparent promising connection, no vacuum state that leads to the details needed for our neutrino model has been discussed to date. In this paper, we will assume the sub-Planck GUT group to be a subgroup of $E_{8} \times E_{8}^{\prime}$ in the hope of possible future string embedding of our model. For alternative theoretical models for the sterile neutrino, see Ref.22.

As suggested in Ref.19, we will assume that the process of spontaneous symmetry breaking introduces asymmetry between the two universes e.g. the weak scale $v_{w k}^{\prime}$ in the mirror universe is larger than the weak scale $v_{w k}=246 \mathrm{GeV}$ in our universe. It was shown in Ref 19 that with this one assumption alone, the gravitationally generated neutrino masses 21 can provide a resolution of the solar neutrino puzzle (i.e. one parameter generates both the required $\Delta m_{e-s}^{2}$ and the mixing angle $\sin ^{2} 2 \theta_{e-s} \simeq$ $\left.10^{-2}\right)$.

\section{An $S O(10) \times S O(10)$ realization of the mirror universe idea}

In a recent paper23, we constructed a complete realistic model for known particles and forces and make detailed numerical predictions for the neutrino sector within a grand unified scheme that implements the seesaw mechanism. Since the simplest GUT model that implements the seesaw mechanism is based on the $S O(10)$, we use SUSY $S O(10) \times S O(10)^{\prime}$ as our gauge group with each $S O(10)$ operating in one sector.

We impose a mirror symmetry 24 between the two $\mathrm{SO}(10)$ sectors so that the field contents as well as the gauge and Yukawa couplings in the two sectors are identical to each other and all differences between them arise from the process of spontaneous symmetry breaking. In order to constrain the model further, we impose the requirement that it conserve R-parity automatically without using any extra global symmetries. This ensures that there is a natural cold dark matter candidate in the model. We also impose an additional global permutation symmetry $S_{3}$ which plays a key role in ensuring the mass degeneracy between the tau and muon neutrinos. The connection between the visible and the mirror sector occurs via the mixing of the heavy right-handed neutrinos 25 .

The nontrivial nature of the problem arises from the fact that in a GUT framework the neutrino couplings are intimately linked to the charged fermion couplings and it is by no means obvious that with a simple set of Higgs fields one can make the observed hierarchical pattern of the charged fermion masses and mixings compatible with the apparent non-hierarchical mass and mixing pattern for the neutrinos.

The fermions of each generation are assigned to the $(\mathbf{1 6}, \mathbf{1}) \oplus\left(\mathbf{1}, \mathbf{1 6}^{\prime}\right)$ representation of the gauge group. We denote them by $\Psi_{e, \mu, \tau}$ in the visible sector and by corresponding symbols with a prime in the mirror sector (as we do for all fields). The $S O(10)$ symmetry is broken down to the left-right symmetric model by the combination of $\mathbf{4 5} \oplus \mathbf{5 4}$ representations in each sector. The $S U(2)_{R} \times U(1)_{B-L}$ gauge symmetry in turn is broken by the $\mathbf{1 2 6} \oplus \overline{\mathbf{1 2 6}}$ representations and we take three such 
representations (and denote them by $\Delta_{0,1,2} \oplus \bar{\Delta}_{0,1,2}$ ). The role of the these fields is two-fold: first, they guarantee automatic R-parity conservation and second, they lead to the see-saw suppression for the neutrino masses26.

The standard model symmetry is then broken by the 10-dim. Higgs fields of which we take three $H_{0,1,2}$. As is well-known, the 126-dim. representation contains in it left-handed triplets with $B-L=2$. Due to the presence of the 54-Higgs field $S$ in the model, couplings of type $\overline{\Delta \Delta} S$ and $H H S$ are allowed and they lead to induced $B-L$ breaking vev's $v_{L}$ which give a direct Majorana mass to the neutrinos leading to the so called type II see saw formula 27 written symbolically as

$$
m_{\nu} \simeq f v_{L}-\frac{m_{\nu^{D}}^{2}}{f v_{R}}
$$

where $v_{R}$ is the generic vev of the $\nu^{c} \nu^{c}$ component of 126. As was shown in 27, the detailed minimization of the potential in such theories leads to the conclusion that $v_{L}$ is also suppressed by a see saw like formula (i.e. $v_{L} \simeq v_{w k}^{2} / \lambda v_{R}$ where $\lambda$ is an unknown parameter in the superpotential). If we choose $v_{R} \simeq 10^{14}-10^{15} \mathrm{GeV}$ (so that it is not far from the GUT scale) and $\lambda \simeq 0.1-0.01$, then we get $v_{L}$ in the $\mathrm{eV}$ range. Note that while the second term in Eq. (3) arising from the conventional see saw formula leads to a hierarchical mass pattern for the neutrinos, the first term has no such obligation. Thus, if we require some neutrinos to be nearly degenerate, the first term has to be given the dominant role as we do here.

A second point is that in the effective MSSM derived from the model, the low energy Higgs doublets will be assumed to be linear combinations of the doublets present in all $\mathbf{1 0}$ as well as $\mathbf{1 2 6}$ dimensional multiplets. In principle this situation can be realized by appropriate arrangement of parameters.

Next we assume the invariance of the action under a discrete permutation symmetry $S_{3}$ under which the $\left(\Psi_{\mu}, \Psi_{\tau}\right),\left(\Delta_{1}, \Delta_{2}\right)$ and $\left(H_{1}, H_{2}\right)$ transform as doublets whereas $\Psi_{e}, H_{0} \Delta_{0}$ and the rest of the fields transform as singlets. The same discrete operates in the mirror sector (i.e. no mirror version of $S_{3}$ ). This then restricts the form of the Yukawa part of the superpotential to the following form:

$$
\begin{array}{r}
W_{Y}=h_{1} \Psi_{e} \Psi_{e} H_{0}+h_{2}\left(\Psi_{\mu} \Psi_{\mu}+\Psi_{\tau} \Psi_{\tau}\right) H_{0}+h_{3} \Psi_{e}\left(\Psi_{\mu} H_{1}+\Psi_{\tau} H_{2}\right) \\
+h_{4}\left[\left(\Psi_{\mu} \Psi_{\mu}-\Psi_{\tau} \Psi_{\tau}\right) H_{1}+2 \Psi_{\mu} \Psi_{\tau} H_{2}\right]+f_{1} \Psi_{e} \Psi_{e} \bar{\Delta}_{0} \\
+f_{2}\left(\Psi_{\mu} \Psi_{\mu}+\Psi_{\tau} \Psi_{\tau}\right) \bar{\Delta}_{0}+f_{3}\left[\left(\Psi_{\mu} \Psi_{\mu}-\Psi_{\tau} \Psi_{\tau}\right) \bar{\Delta}_{1}+2 \Psi_{\mu} \Psi_{\tau} \bar{\Delta}_{2}\right] \\
+f_{4} \Psi_{e}\left(\Psi_{\mu} \bar{\Delta}_{1}+\Psi_{\tau} \bar{\Delta}_{2}\right)
\end{array}
$$

Using Eq. 4, we can write down the quark and lepton mass matrices for the visible sector as follows.

$$
M_{10}^{D}=\left(\begin{array}{ccc}
c_{1} & c_{2} & c_{3} \\
c_{2} & c_{4}+c_{5} & c_{6} \\
c_{3} & c_{6} & c_{4}-c_{5}
\end{array}\right) \quad ; \quad M_{126}^{D}=\left(\begin{array}{ccc}
d_{5} & d_{1} & d_{2} \\
d_{1} & d_{3}+d_{6} & d_{4} \\
d_{2} & d_{4} & d_{6}-d_{3}
\end{array}\right)
$$




$$
\begin{gathered}
M_{10}^{U}=\left(\begin{array}{ccc}
a_{1} & a_{2} & a_{3} \\
a_{2} & a_{4}+a_{5} & a_{6} \\
a_{3} & a_{6} & a_{4}-a_{5}
\end{array}\right) \quad ; \quad M_{126}^{U}=\left(\begin{array}{ccc}
b_{5} & b_{1} & b_{2} \\
b_{1} & b_{3}+b_{6} & b_{4} \\
b_{2} & b_{4} & b_{6}-b_{3}
\end{array}\right) \\
M^{U}=M_{10}^{U}+M_{126}^{U} \quad ; \quad M^{D}=M_{10}^{D}+M_{126}^{D} ; \\
M^{\nu}=M_{10}^{U}-3 M_{126}^{U} \quad ; \quad M^{L}=M_{10}^{D}-3 M_{126}^{D}
\end{gathered}
$$

Even though apriori, it may appear from Eq. 5 and 6 that there are 24 parameters in the visible sector mass matrices, the actual number is 16 due to the $S_{3}$ invariance of the theory which yields eight relations among them. They are $\frac{c_{3}}{c_{6}}=\frac{c_{2}}{c_{5}}=\frac{a_{2}}{a_{5}}=\frac{a_{3}}{a_{6}}$; $\frac{a_{1}}{a_{4}}=\frac{c_{1}}{c_{4}} ; \frac{b_{1}}{b_{3}}=\frac{d_{1}}{d_{3}}=\frac{b_{2}}{b_{4}}=\frac{d_{2}}{d_{4}}$ and $\frac{b_{5}}{b_{6}}=\frac{d_{5}}{d_{6}}$. We now proceed to determine the remaining parameters in such a way they give rise to observed fermion masses and quark mixings and vanishing charged lepton mixings. The best fit is obtained for the following values for standard model parameters at the GUT scale (all masses in GeV units): $m_{t}=112.00, m_{c}=0.370, m_{u}=0.0011 ; m_{b}=1.115, m_{s}=0.0148, m_{d}=0.0013 ; \mathrm{CKM}$ mixing parameters $s_{12}=-0.2201 ; s_{23}=0.031 ; s_{13}=0.0039$ and the lepton masses $m_{e}=0.00033, m_{\mu}=0.0699, m_{\tau}=1.1817$ we find the values for the parameters $a, b, c$, and $d$ listed in table I. They in turn enable us to determine the Dirac neutrino mass matrix for the visible as well as the mirror sector. Although the Dirac mass matrix for the neutrino does not play a significant role in the masses and mixings in the individual sector, we will see in the next section that it plays a crucial role in the mixing between the two sectors.

\section{Connecting the two sectors and predictions for neutrinos}

The neutrino mass matrix has two contributions as is seen from Eq. 3. For $v_{R}$ near $10^{15} \mathrm{GeV}$, the largest entry from the second term in Eq. 1 is of order $10^{-2} \mathrm{eV}$ in the 33 element and much smaller in other places. As far as the first term goes, its form is dictated by Eq. 3 and we choose it as follows:

$$
M_{\nu \nu}=\left(\begin{array}{ccc}
0 & A_{l} & A_{l}^{\prime} \\
A_{l} & B_{l} & D_{l} \\
A_{l}^{\prime} & D_{l} & -B_{l}
\end{array}\right) \quad \text { in } \mathrm{eV}
$$

In order to obtain the predictions for neutrino masses and mixings, we need to know the structure of the mass matrices in the mirror sector and the connection between the visible and the mirror sector.

The exact mirror symmetry between the visible and the mirror sector implies that at the level of the superpotential, all couplings in the mirror sector are identical to those in the visible sector. We will assume that the spontaneous symmetry breaking breaks the mirror symmetry so that actual mass matrices will exhibit differences. For simplicity, we will assume that all doublet vev's in the mirror sector differ by 
a common ratio from those in the visible sector (i.e. $v_{w k}^{\prime} / v_{w k}=\zeta$ ). Since this asymmetry will effect the fermion masses in the two MSSM's, we will expect the $B-L$-breaking scales and the GUT scales to be different. This in turn will imply that the induced triplet vev's will also be different in the two sectors. Our strategy will therefore be to scale the Dirac mass matrix for the mirror sector by a common factor but introduce arbitrary triplet vev's in the mirror sector.

$$
M_{\nu^{\prime} \nu^{\prime}}=\left(\begin{array}{ccc}
\alpha & A_{m} & A_{m}^{\prime} \\
A_{m} & B_{m} & D_{m} \\
A_{m}^{\prime} & D_{m} & -B_{m}
\end{array}\right) \quad \text { in } \mathrm{eV}
$$

Where, $A_{m}=q_{1} A_{l}, A_{m}^{\prime}=q_{1} A_{l}, D_{m}=q_{2} D_{l}$ and $B_{m}=q_{2} B_{l}$. Let us now try to connect the two sectors which we do by postulating the Higgs fields $\left(\mathbf{1 6}, \mathbf{1 6}^{\prime}\right) \oplus\left(\overline{\mathbf{1 6}}, \overline{\mathbf{1 6}}^{\prime}\right)$ (denoted by $\chi \oplus \bar{\chi})$. There can now be a connecting term between the two sectors given by

$$
W^{\prime}=g_{c} \Psi_{e} \Psi_{e}^{\prime} \bar{\chi}+g_{c}^{\prime}\left(\Psi_{\mu} \Psi_{\mu}^{\prime}+\Psi_{\tau} \Psi_{\tau}^{\prime}\right) \bar{\chi}
$$

We now give a vacuum expectation value to the $\nu^{c} \nu^{c \prime}$ element of $\chi \oplus \bar{\chi}$ fields. Then only the right handed neutrinos of both sectors get connected. This in conjunction with the Dirac masses of both sectors introduces a mixing matrix between the two sectors of the following form (assuming for simplicity $g_{c}=g_{c}^{\prime}$ ):

$$
M_{\nu \nu^{\prime}}=g_{c} M_{\nu^{D}} M_{\nu^{c}}^{-2} M_{\nu^{D}}<\chi>
$$

Where,

$$
M_{\nu^{c} \nu^{c}}=\left(\begin{array}{ccc}
0 & A_{r} & A_{r}^{\prime} \\
A_{r} & B_{r} & D_{r} \\
A_{r}^{\prime} & D_{r} & -B_{r}
\end{array}\right) \quad \text { in } \mathrm{GeV}
$$

Where, $A_{r}=l_{1} A_{l}, A_{r}^{\prime}=l_{1} A_{r}^{\prime}, B_{r}=l_{2} B_{l}$ and $D_{r}=l_{2} D_{l}$. We diagonalize the complete neutrino mass matrix to obtain the following absolute values of the mass eigenvalues (in eV's): $m_{\nu_{\tau}^{\prime}}=90.56, m_{\nu_{\mu}^{\prime}}=-90.56, m_{\nu_{e}^{\prime}}=0.0034 m_{\nu_{\tau}}=1.51, m_{\nu_{\mu}}=$ $-1.509, m_{\nu_{e}}=0.001$. The squared mass diffences (in $\mathrm{eV}^{2}$ ) are $\Delta m_{e-s}^{2}=9.9 \times 10^{-6}$, $\Delta m_{\mu-\tau}^{2}=0.003$ and $\Delta m_{e-\mu}^{2}=2.27$, where the numbers are given in $\mathrm{eV}^{2}$. The fitted values of the parameters are, $\alpha=0.005, A_{l}=0.0253, A_{l}^{\prime}=0.050, B_{l}=0.675$, $D_{l}=1.35$ given in eV units, $q_{1}=10, q_{2}=60, l_{1}=1.210^{15}, l_{2}=0.510^{15}$ and $g_{c}<\chi>=6.9 \times 10^{12}$ given in $\mathrm{GeV}$ units. The mixing matrix $O^{\nu}$ of the six neutrinos in the basis $\left(\nu_{e}, \nu_{\mu}, \nu_{\tau}, \nu_{e}^{\prime}, \nu_{\mu}^{\prime}, \nu_{\tau}^{\prime}\right)$ is approximately given as,

$$
O^{\nu}=\left(\begin{array}{cccccc}
-0.99 & 0.037 & 0 & 0.039 & -0.00025 & 0 \\
-0.031 & -0.85 & -0.52 & -0.00072 & 0 & 0 \\
0.019 & 0.525 & -0.85 & -0.00043 & 0 & 0 \\
-0.042 & 0.0014 & 0.00071 & -0.999 & 0.0062 & 0 \\
0 & 0 & 0 & -0.0053 & -0.850 & -0.525 \\
0 & 0 & 0 & 0.0032 & 0.52 & -0.85
\end{array}\right)
$$


Combining this with the mixing angle for the charged leptons, we obtain the final mixing matrix among the four neutrinos which looks identical to the corresponding top-left $4 \times 4$ submatrix of $O_{\nu}$ with only the $\nu_{e}-\nu_{\tau}$ entry reduced by a factor of 2 because of the presence of a small 13 element in the charged leptonic mass matrix. We have varied the parameters of the model to see the allowed range for the $m_{\nu_{\mu}}$ relevant for the LSND experiment and find consistent solutions for the range $0.5 \leq$ $m_{\nu_{\mu}} / e V \leq 1.5$. Therefore, the $\nu_{\mu}$ and $\nu_{\tau}$ together could play the role of the hot dark matter for the upper allowed range of the masses. Also note that the zeros in the neutrino mixing matrix simply means that those entries are less than $10^{-4}$.

We thus see that in this model not only are all three positive indications of neutrino oscillations are explained but the mixing between the heavier sterile neutrinos $\nu_{\mu}^{\prime}$ and $\nu_{\tau}^{\prime}$ and the active neotrinos are consistent with all known oscillation data such as for example the one from the CHOOZ28. What we find very interesting is that with only six parameters describing the entire $6 \times 6$ neutrino mass matrix (three active and three sterile) and every other parameter fixed by the charged fermion masses, four neutrino masses and 12 mixing parameters that link the active to sterile neutrinos which could have observable consequences are all completely consistent with known data.

Turning now to the consistency of our model with big bang nucleosynthesis (BBN), we recall that present observations of Helium and deuterium abundance can allow for as many as 4.53 neutrino speciest if the baryon to photon ratio is small. In our model, since the neutrinos decouple above $200 \mathrm{MeV}$ or so, their contribution at the time of nucleosynthesis is negligible (i.e. they contribute about 0.3 to $\Delta N_{\nu}$.) On the other hand the mirror photon could be completely in equilibrium at $T=1 \mathrm{MeV}$ so that it will contribute $\Delta N_{\nu}=1.11$. All together the total contribution is les than 1.5.

In conclusion, if the LSND result stands the test of time, this would indicate an ultralight sterile neutrino. An interesting model for understanding its lightness is to postulate a mirror universe. This can be converted into a fully grand unified model within the $S O(10) \times S O(10)$ group.

I am grateful to D. Caldwell, Z. Berezhiani and B. Brahmachari for collaboration and discussions on the sterile neutrino idea. I am also very grateful to B. Kniehl and G. Raffelt for kind hospitality at the Ringberg castle. This work is supported by the National Science Foundation under grant no. PHY-9802551.

1. T. Kajita, Invited talk at the Neutrino98, Takayama, Japan (to appear in the proceedings); Super-Kamiokande Collaboration, UMD-PP-98- 117 and ICRR Report-411-98-7.

2. B. T. Cleveland et al. Nucl. Phys. B(Proc. Suppl.) 38, 47 (1995); K.S. Hirata et al., Phys. Rev. 44, 2241 (1991); GALLEX Collaboration, Phys. Lett. B388, 384 (1996); J. N. Abdurashitov et al., Phys. Rev. Lett. 77, 4708 (1996).

3. Y. Suzuki, Invited talk at Erice Neutrino workshop, September 17-22, 1997. 
4. K.S. Hirata et al., Phys. Lett. B280, 146 (1992); R. Becker-Szendy et al., Phys. Rev. D 46, 3720 (1992); W. W. M. Allison et al., Phys. Lett. B 391, 491 (1997); Y. Fukuda et al, Phys. Lett. B 335, 237 (1994).

5. C. Athanassopoulos et al. Phys. Rev. Lett. 75, 2650 (1995); LSND2 C. Athanassopoulos et al. nucl-ex/9706006.

6. D.O. Caldwell and R.N. Mohapatra, Phys. Rev. D 48, 3259 (1993); J. Peltoniemi and J. W. F. Valle, Nucl. Phys. B 406, 409 (1993).

7. S. Bilenky, C. Giunti and W. Grimus, Eur. Phys. J. C 1, 247 (1998); hepph/9805368; S. Goswami, Phys. Rev. D 55, 2931 (1997); N. Okada and O. Yasuda, hep-ph/9606411.

8. R. Foot and R. Volkas, Phys. Rev. D52, 6595 (1995).

9. Q. Liu and A. Smirnov, hep-ph/9712493.

10. S. P. Mikheyev and A. Smirnov, Sov. J. Nucl. Phys. 42, 913 (1985); L. Wolfenstein, Phys. Rev. D 17, 2369 (1978).

11. E. Gawiser and J. Silk, astro-ph/9806197; Science, 280, 1405 (1998).

12. B. Armbruster et al., hep-ex/9801007.

13. SNO collaboration, E. Norman et al., "Proceedings of The Fermilab Conference: DPF 92 ed. by C. Albright, P. H. Kasper, R. Raja and J. Yoh (World Scientific), p. 1450.

14. A. Smirnov and F. Vissani, hep-ph/9710565; J. G. Learned, S. Pakvasa and J. Stone, hep-ph/9805343.

15. V. Barger, K. Whisnant and T. Weiler, hep-ph/9712495; S. Gibbons, R. N. Mohapatra, S. Nandi and A. Raichoudhuri, hep-ph/9803299 (to appear in Phys. Lett. B).

16. D. Caldwell, G. Fuller and Y. Qian, to appear.

17. S. Sarkar, Rep. Prog. Phys. 59, 1493 (1996); P. Kernan and S. Sarkar, Phys. Rev. D 54, 3681 (1996).

18. R. Barbieri and A. Dolgov, Phys. Lett. B 237, 440 (1990); K. Enquist, K. Kainulainen and J. Maalampi, Phys. Lett. B 249, 531 (1992); D. P. Kirilova and M. Chizov, hep-ph/9707282.

19. Z. Berezhiani and R. N. Mohapatra, Phys. Rev. D 52, 6607 (1995).

20. S. I. Blinikov and M. Yu Khlopov, Sov. Astron. 27, 371 (1983); Z. Silagadze, hep-ph/9503481.

21. R. Barbieri, J. Ellis and M. K. Gaillard, Phys. Lett. 90 B, 249 (1980); E. Akhmedov, Z. Berezhiani and G. Senjanović, Phys. Rev. Lett. 69, 3013 (1992).

22. K. Benakli and A. Smirnov, Phys. Rev. Lett. 79, 4314 (1997); P. Langacker, hep-ph/9805281; D. Suematsu, Phys. Lett. B392, 413 (1997); E. J. Chun, A. Joshipura and A. Smirnov, Phys. Rev. D 54, 4654 (1996); E. Ma and P. Roy, Phys. Rev. D 52, 4342 (1995); M. Bando and K. Yoshioka, hep-ph/9806400; N. Arkani-Hamed and Y. Grossman, hep-ph/9806223. 
23. B. Brahmachari and R. N. Mohapatra, hep-ph/9805429.

24. R. Foot, H. Lew and R. Volkas, Phys. Lett. B272, 67 (1991).

25. M. Collie and R. Foot, hep-ph/9803261.

26. M. Gell-Mann, P. Ramond and R. Slansky, in Supergravity, eds. P. van Niewenhuizen and D.Z. Freedman (North Holland 1979); T. Yanagida, in Proceedings of Workshop on Unified Theory and Baryon number in the Universe, eds. O. Sawada and A. Sugamoto (KEK 1979); R. N. Mohapatra and G. Senjanović, Phys. Rev. Lett. 44, 912 (1980).

27. R. N. Mohapatra and G. Senjanović, Phys. Rev. D23, 165 (1981).

28. M. Appollonio et al. hep-ex/9711002.

\begin{tabular}{|c||c||c||c||c||c|}
\hline$a_{1}$ & $a_{2}$ & $a_{3}$ & $a_{4}$ & $a_{5}$ & $a_{6}$ \\
0.140 & 0.230 & 0 & 50.54 & -55.30 & 0 \\
\hline \hline$c_{1}$ & $c_{2}$ & $c_{3}$ & $c_{4}$ & $c_{5}$ & $c_{6}$ \\
0.0016 & 0.0023 & -0.00012 & 0.598 & -0.55 & 0.0288 \\
\hline$b_{1}$ & $b_{2}$ & $b_{3}$ & $b_{4}$ & $b_{5}$ & $b_{6}$ \\
0.140 & 0.230 & 0 & 50.54 & -55.30 & 5.64 \\
\hline \hline$d_{1}$ & $d_{2}$ & $d_{3}$ & $d_{4}$ & $d_{5}$ & $d_{6}$ \\
0.0016 & 0.0023 & -0.00012 & 0.598 & -0.55 & -0.0152 \\
\hline
\end{tabular}

Table 1: The fitted values of $a_{i}, b_{i}, c_{i}$ and $d_{i}$ 